\title{
Mechanism of theophylline-induced inotropic effects on foreshortened canine diaphragm
}

\author{
G. Gayan-Ramirez*, S. Janssens*, B. Himpens**, M. Decramer*
}

Mechanism of theophylline-induced inotropic effects on foreshortened canine diaphragm. G. Gayan-Ramirez, S. Janssens, B. Himpens, M. Decramer. @ERS Journals Ltd 1995. ABSTRACT: The mechanisms of theophylline-induced inotropic effects at shorter diaphragm length have not yet been explored. We wondered whether the greater inotropic effects of the drug at shorter diaphragm length might result from an effect on intracellular calcium level.

Forty pairs of diaphragm bundles were stimulated at $70 \%$ of optimal length in the presence of either verapamil $\left(1^{-5} \mathrm{M}\right)$, calcium-free Krebs solution (buffered or not with $2 \mathrm{mM}$ ethylene glycol tetra-acetic acid (EGTA)) or ryanodine $\left(10^{-6} \mathrm{M}\right)$. Theophylline $(1 \mathrm{mM})$ was subsequently added to one muscle bundle and, after 15 min, twitches were repeated.

The twitch potentiation induced by theophylline $(37 \pm 21 \%)$ was unaffected by verapamil $(43 \pm 26 \%)$, or zero calcium $(39 \pm 18 \%)$ and virtually unchanged when the latter was buffered with EGTA. By contrast, theophylline failed to increase twitch tension after pretreatment with ryanodine, a blocker of the calcium release by the sarcoplasmic reticulum. This decreased twitch tension in control $(-5 \pm 11 \%)$ and experimental $(-14 \pm 12 \%)$ bundles and prolonged half-relaxation time as a result of impaired sarcoplasmic reticulum calcium reuptake.

We conclude that the inotropic effects of theophylline on twitch tension in foreshortened canine diaphragm bundles were not related to transmembrane calcium flux but were dependent on the release of calcium from the sarcoplasmic reticulum. This is consistent with an action of theophylline on the sarcoplasmic reticulum. Eur Respir J., 1995, 8, 1915-1921.

\begin{abstract}
*Respiratory Muscle Research Unit, Laboratory of Pneumology, Respiratory Division and **Laboratory of Physiology, Katholieke Universiteit Leuven, Leuven, Belgium.
\end{abstract}

Correspondence: M. Decramer

Respiratory Division

University Hospital

Herestraat 49

B-3000 Leuven

Belgium

Keywords: Contractile properties respiratory muscles

theophylline and calcium

Received: September 21994

Accepted after revision August 41995

Supported by the "Fonds voor Geneeskundig Wetenschappelijk Onderzoek".
In a previous in vivo study, we demonstrated that theophylline exerted more pronounced inotropic effects on foreshortened ( $70 \%$ of optimal length) canine diaphragm than on diaphragm placed at optimal length [1]. This difference was greater at low than at high stimulation frequencies and increased with increasing theophylline concentration. The preferential inotropic effect on foreshortened diaphragm was subsequently confirmed in vitro on canine diaphragm [2], showing, thereby, that the enhanced inotropic effects essentially resulted from an effect on the muscle itself and not from changes in rib cage or abdominal compliances, or diaphragm geometry.

The mechanism of theophylline-induced inotropic effects at shorter length has never been explored. To investigate this mechanism, the alterations in muscle mechanics with shortening need to be considered in order to demonstrate the potential action site of theophylline. Thus, the decrease in filament overlap between actin and myosin when muscle shortens [3] does not fully account for the decrease in tension generation observed at shorter muscle length. Indeed, due to muscle shortening, fibres are swollen, thereby compressing the T-tubular systems and "impeding exit electrolyte flow" as described previously [4]. Therefore, impairment of T-tubular conduction and/or hampering of calcium release from the sarcoplasmic reticulum may occur because of insufficient signal transmission between T-tubular system and terminal cisternae $[4,5]$. As a consequence, the action potential may not reach the central part of the muscle. This relates acute muscle shortening to calcium "deactivation" and probably explains that, at shorter muscle length, the central myofibrils of the muscle are not activated [6], the shortening velocity of central sarcomeres is decreased [7], and the rise in intracellular calcium is less [5].

Therefore, keeping in mind the results of the abovementioned studies, we hypothesized that the greater inotropic effects of theophylline at shorter diaphragm length might result from an effect on intracellular calcium level. Consequently, each pathway able to enhance cytoplasmic calcium was selectively blocked and the effects of theophylline on twitch tension of foreshortened canine diaphragm examined in vitro under each condition.

\section{Methods}

\section{Experimental set-up}

Sixteen mongrel dogs $(14.9 \pm 1.8 \mathrm{~kg})$ were anaesthetized with pentobarbital sodium (Nembutal, initial dose 
$25 \mathrm{mg} \cdot \mathrm{kg}^{-1}$ i.v.) and mechanically ventilated (Hospal 251). According to Ethics Committee guidelines, animals did not experience any suffering. The level of anaesthesia was regulated to abolish the corneal reflex. After a midline laparotomy, a segment of costal diaphragm (width ranging $2-3 \mathrm{~cm}$ ) was removed and immediately immersed in a cooled oxygenated Krebs solution for further dissection of two small bundles. For each dog, six pairs of diaphragm bundles were obtained. These bundles were then placed within the external chamber of a jacketed tissue bath filled with normal Krebs solution maintained at $37^{\circ} \mathrm{C}$ and perfused with $95 \% \mathrm{O}_{2}$ and $5 \% \mathrm{CO}_{2}$, $\mathrm{pH}$ 7.3-7.4. The Krebs solution contained in mM: 137 $\mathrm{NaCl}, 4 \mathrm{KCl}, 2 \mathrm{CaCl}_{2}, 1 \mathrm{MgCl}_{2}, 1 \mathrm{KH}_{2} \mathrm{PO}_{4}, 12 \mathrm{NaHCO}_{3}$, and 6.5 glucose. One end of the bundle was attached to a rigid support whilst the other was fastened to an isometric force transducer mounted to a micrometer. Two large platinum stimulating electrodes were placed on both sides of the muscle bundle.

\section{General procedure}

After a 15 min thermoequilibration period, each bundle was placed at optimal length (Lo), defined as the length at which peak twitch force was obtained. Stimulation was delivered through a Harvard 50-5016 stimulator (Edenbridge, Kent, UK) which was connected in series to a power amplifier made from power one model HS244.8 developed by the computer technology resources centre, University of Virginia, USA (R.J. Evans, 1983).
Maximal stimulation was achieved at approximately $34 \mathrm{~V}$. The voltage was then increased by $20 \%$ to ensure supramaximal stimulation. Isometric force was measured with a Maywood force transducer (Maywood Ltd, Hampshire, UK). The signal was amplified and recorded on computer via analog-to-digital conversion (DT 2801-A) using Labdat (Labdat/Anadat, RHT-Infodat, Montreal, Canada). Signal analysis was performed with anadat.

Two twitches and one tetanic stimulation $(100 \mathrm{~Hz}$ applied during $450 \mathrm{~ms}$ with square waves of $0.5 \mathrm{~ms}$ ) were recorded at Lo. Subsequently, both bundles were shortened to $70 \%$ Lo and stimulated twice at $1 \mathrm{~Hz}$ after $2 \mathrm{~min}$. For twitches, time to peak tension (TPT) and half-relaxation time (1/2RT) were also measured.

Different protocols were then followed (tables 1 and 2) in which two bundles were studied simultaneously, eight pairs for each protocol.

\section{Experimental protocols}

The present study first examined the effects of a single dose of theophylline on twitch tension. Theophylline was used at a concentration of $1 \mathrm{mM}$, a concentration previously shown to produce clear inotropic effects on foreshortened diaphragm [2]. When used, theophylline was added to one bundle within a given pair $15 \mathrm{~min}$ before monitoring its influence on twitch tension (table 1). At the same time, the other bundle received normal Krebs solution to serve as time-matched control (table 1).

Table 1. - Summary of experimental protocols with calcium-free Krebs solution $\left(0 \mathrm{Ca}^{2+}\right)$ buffered or not with EGTA and verapamil

\begin{tabular}{l}
$\begin{array}{l}\text { Incubation } \\
\text { time } \\
\text { min }\end{array}$ \\
\hline 15
\end{tabular}

Table 2. - Summary of experimental protocols with ryanodine

\begin{tabular}{|c|c|c|c|c|c|}
\hline \multirow{2}{*}{$\begin{array}{l}\text { Incubation } \\
\text { time } \\
\text { min } \\
40\end{array}$} & \multirow{2}{*}{\multicolumn{3}{|c|}{$\begin{array}{c}\begin{array}{c}\text { Incubation } \\
\text { time } \\
\text { min }\end{array} \\
15\end{array}$}} & \multicolumn{2}{|c|}{ Krebs/ryanodine } \\
\hline & & & & $\begin{array}{c}\text { Krebs } \\
2 P_{\mathrm{t}}\end{array}$ & $\begin{array}{c}\text { Theophylline } \\
2 P \mathrm{t}\end{array}$ \\
\hline 15 & $\begin{array}{c}\underset{\nabla}{\text { Ryanodine }} \\
2 P \mathrm{t}\end{array}$ & $\begin{array}{c}\text { Theophylline } \\
2 P \mathrm{t}\end{array}$ & 40 & $\begin{array}{c}\underset{\text { Krebs }}{\nabla} \\
2 P_{\mathrm{t}}\end{array}$ & $\begin{array}{c}\text { Ryanodine } \\
2 P \mathrm{t}\end{array}$ \\
\hline
\end{tabular}

Pt: twitch tension. 
It should be noted that, in this protocol, bundles were first incubated for $15 \mathrm{~min}$ with normal Krebs solution (table 1) to synchronize timing with the following protocols.

The effects of calcium channel blockers and zero calcium on theophylline-induced twitch potentiation were subsequently evaluated in separate protocols. Verapamil (Isoptine $2.5 \mathrm{mg} \cdot \mathrm{mL}^{-1}$; Knoll, Germany) was used to block calcium entry, whereas ryanodine (produced from a $10^{-2} \mathrm{M}$ solution; Molecular Probes, Oregon, USA) inhibited the release of calcium from the sarcoplasmic reticulum. In these protocols, blocking concentrations of either verapamil $10^{-5} \mathrm{M}$ or ryanodine $10^{-6} \mathrm{M}$ were used. These agents were added to bundles $15 \mathrm{~min}$ (verapamil) or $40 \mathrm{~min}$ (ryanodine) before theophylline (tables 1 and 2 ). Note that the effects of ryanodine on twitch tension were examined before adding theophylline (table 2), as ryanodine was previously shown to depress twitch tension $[8,9]$.

In addition, a supplemental protocol was performed with ryanodine to ensure the nontoxicity of this agent. In this protocol, theophylline was first given and then ryanodine while, at the same time, Krebs solution alone was added to the other bundles to serve as time-matched controls (table 2).

Finally, for experiments performed in the absence of calcium, normal Krebs solution was replaced by a calcium-free Krebs solution $15 \mathrm{~min}$ prior to the addition of theophylline. The same experiments were repeated with a calcium-free Krebs solution buffered with $2 \mathrm{mM}$ ethylene glycol-bis( $\beta$-amino-ethyl-ether) N, N, N', N'tetra-acetic acid (EGTA), in order to minimize extracellular calcium (either still present in the solution or located in the transverse tubular system).

\section{Data analysis}

At the end of each experiment, the length, thickness and width of each bundle were measured at Lo, and

Table 3. - Contractile properties of the bundles at optimal length (Lo) and at 70\% Lo under control conditions

\begin{tabular}{lccccc}
\hline & $\begin{array}{c}P \mathrm{t} \\
\mathrm{N} \cdot \mathrm{cm}^{-2}\end{array}$ & $\begin{array}{c}\mathrm{TPT} \\
\mathrm{ms}\end{array}$ & $\begin{array}{c}1 / 2 \mathrm{RT} \\
\mathrm{ms}\end{array}$ & $\begin{array}{c}P_{\mathrm{o}} \\
\mathrm{N} \cdot \mathrm{cm}^{-2}\end{array}$ & $P \mathrm{t} / P_{\mathrm{o}}$ \\
\hline Lo & $7.6 \pm 1.7$ & $43 \pm 9$ & $62 \pm 9$ & $23 \pm 3$ & $0.34 \pm 0.06$ \\
$70 \% \mathrm{Lo}$ & $3.0 \pm 1.2$ & $30 \pm 8$ & $47 \pm 9$ & - & - \\
\hline
\end{tabular}

Pooled values of twitch tension $(P \mathrm{t})$, time to peak tension (TPT), half-relaxation time $(1 / 2 \mathrm{RT})$, tetanic tension $\left(P_{\mathrm{o}}\right)$ and twitch to tetanus ratio $\left(P_{\mathrm{t}} / P_{\mathrm{o}}\right)$. Notice that at $70 \% \mathrm{Lo}, P_{\mathrm{o}}$ was not measured so that $P \mathrm{t} / P_{\mathrm{o}}$ cannot be calculated. bundles were weighed. Cross-sectional area (CSA) was calculated by dividing weight by specific density (1.056) and muscle length. Twitch and tetanic forces were expressed per unit cross-sectional area.

Data of theophylline-treated bundles were compared to data obtained with calcium blockers or zero calcium using two-way analysis of variance. A p-value of less than 0.05 was set as the level of significance. Differences between means were assessed by a subsequent Duncan test. Data reported in the text and figures are means \pm standard deviation (SD).

\section{Results}

Geometric and contractile properties of the bundles at Lo

Geometric properties of the bundles were similar at Lo for each protocol. For all studies combined $(n=96)$, length, thickness, weight and cross-sectional area obtained at Lo averaged $4.3 \pm 0.8 \mathrm{~cm}, 1.4 \pm 0.4 \mathrm{~mm}, 0.15 \pm 0.05 \mathrm{~g}$ and $0.036 \pm 0.029 \mathrm{~cm}^{2}$, respectively. Similarly, contractile properties of bundles were the same at Lo in all protocols. Pooled values $(n=96)$ of twitch tension $(P t)$, time to peak tension, half-relaxation time, tetanic tension, and twitch to tetanus ratio are summarized in table 3 . These values are in the range of that reported at $37^{\circ} \mathrm{C}$ in dog diaphragm [10].

\section{Mechanisms of inotropic effects at 70\% Lo}

Twitch characteristics. Under control conditions (before addition of drugs), the pooled values of $P \mathrm{t}$, TPT and $1 / 2 \mathrm{RT}$ obtained at $70 \%$ Lo are summarized in table 3 $(n=96)$. In a given protocol, no statistically significant differences in twitch characteristics were observed between the pairs except for the protocol evaluating the effect of theophylline $(1 \mathrm{mM})$. Indeed, in the latter $(\mathrm{n}=16), P \mathrm{t}$ was higher in the time-matched control bundles than in theophylline-treated bundles $(3.9 \pm 0.7 v s$ $\left.2.9 \pm 1.0 \mathrm{~N} \cdot \mathrm{cm}^{-2} ; \mathrm{p}<0.01\right)$ but TPT and $1 / 2 \mathrm{RT}$ were similar in the two groups (table 4).

Effects of theophylline $(1 \mathrm{mM})$ on twitch tension. In time-matched controls, $P$ tended to decrease by $12 \pm 9 \%$, while $P \mathrm{t}$ of theophylline-treated bundles increased significantly by $37 \pm 21 \%(\mathrm{p}<0.01)$ (fig. 1 ). TPT tended to increase after theophylline and 1/2RT was significantly

Table 4. - Time to peak tension (TPT) and half-relaxation time (1/2RT) of diaphragm bundles at $70 \%$ optimal length (70\%Lo) for each protocol described in table 1

\begin{tabular}{|c|c|c|c|c|c|c|c|c|c|}
\hline \multirow[b]{2}{*}{ TPT } & \multirow[b]{2}{*}{ Before } & \multicolumn{2}{|c|}{ Krebs/theophylline } & \multicolumn{2}{|c|}{$0 \mathrm{Ca}^{2+} /$ theophylline } & \multicolumn{2}{|c|}{ 0 $\mathrm{Ca}^{2+}+\mathrm{EGTA} /$ theophylline } & \multicolumn{2}{|c|}{ Verapamil/theophylline } \\
\hline & & $31 \pm 6$ & $31 \pm 10$ & $26 \pm 8$ & $34 \pm 19$ & $29 \pm 10$ & $26 \pm 12$ & $29 \pm 13$ & $34 \pm 13$ \\
\hline & After & $32 \pm 5$ & $36 \pm 19$ & $36 \pm 19$ & $36 \pm 12$ & $27 \pm 16$ & $28 \pm 11$ & $32 \pm 9$ & $38 \pm 13$ \\
\hline \multirow[t]{2}{*}{$1 / 2 \mathrm{RT}$} & Before & $46 \pm 6$ & $48 \pm 8$ & $46 \pm 7$ & $51 \pm 7$ & $42 \pm 10$ & $45 \pm 11$ & $52 \pm 14$ & $51 \pm 6$ \\
\hline & After & $49 \pm 6$ & $60 \pm 10^{*}$ & $49 \pm 7$ & $52 \pm 6$ & $45 \pm 8$ & $50 \pm 18$ & $57 \pm 15$ & $56 \pm 10$ \\
\hline
\end{tabular}

Values are expressed in ms. *: $\mathrm{p}<0.01$ vs respective control (before). For definitions see legend to tables 1 and 2. 


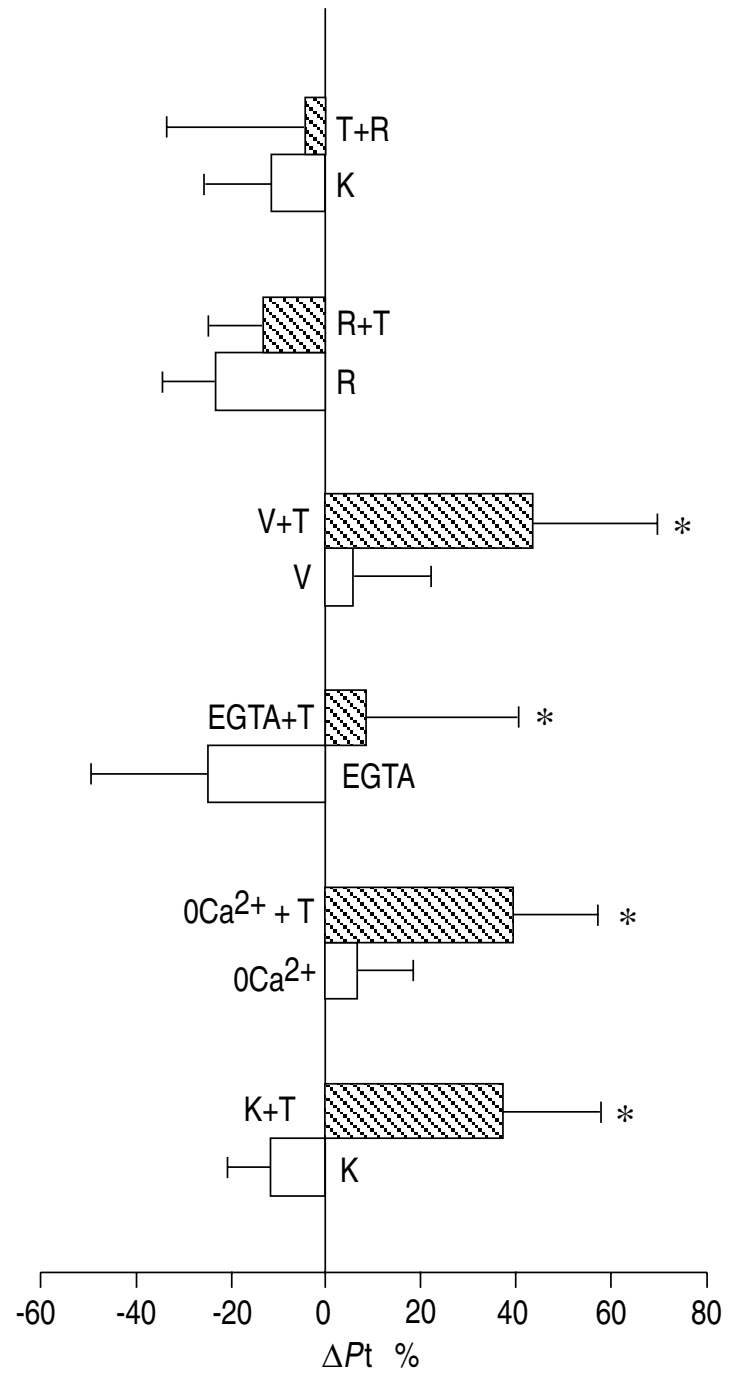

Fig. 1. - Effects of calcium blockers and calcium-free Krebs solution on theophylline-induced twitch potentiation in foreshortened canine diaphragm bundles. Changes in $P_{\mathrm{t}}(\Delta \mathrm{Pt})$ are expressed as percentage of initial $P_{\text {t. }} \quad P_{\text {t: }}$ twitch tension; K: normal Krebs solution; T: theophylline $1 \mathrm{mM}$; $0 \mathrm{Ca}^{2+}$ : calcium-free Krebs solution; EGTA: calciumfree Krebs solution buffered with $2 \mathrm{mM}$ ethylene glycol-bis ( $\beta$-amino-ethyl ether) N, N, N', N'-tetra acetic acid (EGTA); V: verapamil $10^{-5} \mathrm{M}$; $\mathrm{R}$ : ryanodine $10^{-6} \mathrm{M}$. For each protocol, eight pairs of diaphragm bundles were studied; *: $\mathrm{p}<0.05$ experimental (hatched bars) vs control (open bars) bundles. prolonged $(\mathrm{p}<0.01)$ (table 4$)$. In the control bundles, TPT and 1/2RT remained unchanged (table 4).

Effects of zero calcium on theophylline-induced effects at $70 \%$ Lo. Compared to values obtained before treatment, $P \mathrm{t}$ of the bundles treated with both zero calcium and theophylline increased significantly by $39 \pm 18 \%$ $(\mathrm{p}<0.001)$, while $P \mathrm{t}$ of the bundles with zero calcium remained stable (fig. 1). TPT and 1/2RT were unaffected by any treatment (table 4 ).

In bundles incubated with zero calcium buffered with $2 \mathrm{mM}$ EGTA, $P$ t decreased significantly by $25 \pm 25 \%$ $(\mathrm{p}<0.05)$, such that after EGTA pretreatment the increase in $P \mathrm{t}$ of theophylline-treated bundles was only $8 \pm 32 \%$ (fig. 1). The difference between the $P \mathrm{t}$ of theophyllinetreated bundles and that of time-matched controls, however, remained in the range of the expected effect of theophylline on $P \mathrm{t}$. TPT and 1/2RT were unaltered under these conditions (table 4).

Effects of calcium entry blockade on theophylline inotropic effects at $70 \%$ Lo. Whilst $P$ t significantly increased by $43 \pm 26 \%$ after incubation with verapamil $\left(10^{-5} \mathrm{M}\right)$ and theophylline $(\mathrm{p}<0.01)$, it remained unchanged after treatment with verapamil alone (fig. 1). TPT and 1/2RT remained unchanged whatever the treatment (table 4 ).

Effects of calcium release blockade on theophylline inotropic effects at $70 \% \mathrm{Lo}$. In all bundles, ryanodine tended to induce a slight decrease in $P \mathrm{t}$ of $13 \pm 18 \%$ for bundles treated only with ryanodine during the whole protocol, and of $10 \pm 18 \%$ for bundles subsequently treated with theophylline. This decrease did not reach statistical significance and was associated with a prolonged 1/2RT (table 5) as observed previously [8,9]. In addition, the passive tension was progressively increased after ryanodine treatment in both groups (from $52 \pm 59$ to $72 \pm 65$ and from $38 \pm 42$ to $56 \pm 35 \mathrm{mN}$, respectively). The subsequent addition of theophylline failed to increase $P \mathrm{t}$, which remained depressed (fig. 1). Baseline tension further increased to $67 \pm 45 \mathrm{mN}$ after theophylline, whilst it remained similar in the other bundles. 1/2RT remained longer in both groups (table 5). Figure 2a shows an example of twitch profiles obtained in a theophyllinetreated bundle before any treatment and after ryanodine and subsequent addition of theophylline.

Table 5. - Time to peak tension (TPT) and half-relaxation time (1/2RT) of diaphragm bundles at $70 \%$ optimal length $(70 \%$ Lo) for each protocol with ryanodine described in table 2

\begin{tabular}{|c|c|c|c|c|c|}
\hline \multirow[b]{2}{*}{ TPT } & \multirow[b]{2}{*}{ Before } & \multicolumn{2}{|c|}{ Ryanodine/theophylline } & \multicolumn{2}{|c|}{ Krebs/ryanodine } \\
\hline & & $28 \pm 6$ & $30 \pm 5$ & $29 \pm 7$ & $31 \pm 5$ \\
\hline & After & $27 \pm 6(\mathrm{R})$ & $28 \pm 5$ & $29 \pm 12(\mathrm{~K})$ & $34 \pm 5$ \\
\hline & & $25 \pm 7(\mathrm{R})$ & $(\mathrm{R}+\mathrm{T})$ & $32 \pm 7 \quad(\mathrm{~K})$ & $28 \pm 5 \quad(\mathrm{~T}+\mathrm{R})$ \\
\hline \multirow[t]{3}{*}{$1 / 2 \mathrm{RT}$} & Before & $46 \pm 8$ & $43 \pm 8$ & $45 \pm 8$ & $49 \pm 10$ \\
\hline & After & $53 \pm 6(\mathrm{R})$ & $56 \pm 8 * \quad(\mathrm{R})$ & $48 \pm 7 \quad(\mathrm{~K})$ & $53 \pm 6 \quad(\mathrm{~T})$ \\
\hline & & $54 \pm 9(\mathrm{R})$ & $51 \pm 10 *(\mathrm{R}+\mathrm{T})$ & $51 \pm 10(\mathrm{~K})$ & $54 \pm 15(\mathrm{~T}+\mathrm{R})$ \\
\hline
\end{tabular}

Values are expressed in ms. *: $\mathrm{p}<0.01$ vs respective control (before). R: ryanodine $10^{-6} \mathrm{M}$; T: theophylline $1 \mathrm{mM}$; K: normal Krebs solution. 
a)

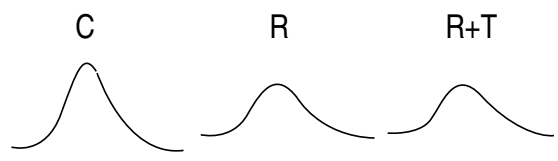

b)

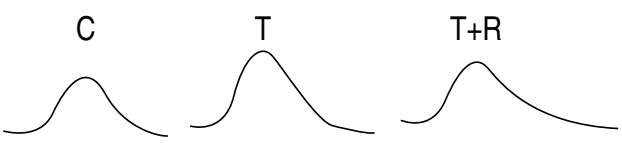

c)

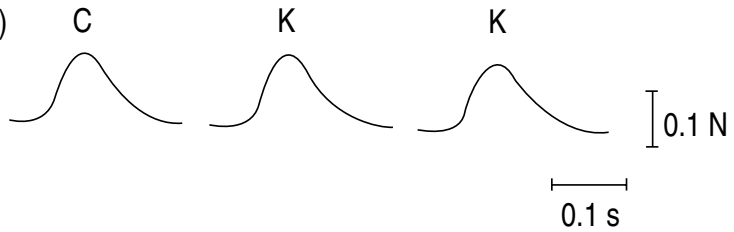

Fig. 2. - Representative tracing showing: a) the twitch depressant effect of ryanodine $(R)$ blocking the inotropic effect of theophylline $(\mathrm{R}+\mathrm{T})$; b) the reversal of theophylline-induced rise (T) in twitch tension by ryanodine $(\mathrm{T}+\mathrm{R})$; and c) twitches of time-matched control bundle. This bundle was treated with normal Krebs solution $(\mathrm{K})$ given at the equivalent time-points. C: control twitch obtained before treatment.

In the protocol evaluating a possible toxic effect of ryanodine, theophylline significantly increased $P$ t by $31 \pm$ $27 \%(\mathrm{p}<0.01)$, while TPT and $1 / 2 \mathrm{RT}$ remained unchanged (table 5). Moreover, passive tension was slightly increased after theophylline (from $28 \pm 40$ to $34 \pm 43 \mathrm{mN}$ ). At the same time, $P \mathrm{t}$ of the Krebs-treated bundles did not change, neither did TPT, 1/2RT (table 5) nor baseline or passive tension $(46 \pm 46 v s 44 \pm 45 \mathrm{mN})$.

The subsequent addition of ryanodine reversed the theophylline-induced increase in $P$ t (fig. 1). This effect was associated with a further and greatly increased passive tension (from $34 \pm 43$ to $67 \pm 57 \mathrm{mN}$ ). At the same time, $P \mathrm{t}$ of the time-matched control bundles tended to decrease by $12 \pm 14 \%$ (fig. 1 ), with no significant changes in TPT and 1/2RT (table 5). Figure $2 \mathrm{~b}$ and c depicts an example of a twitch tracing recorded in a theophylline-treated bundle (fig. 2b) before and after theophylline and subsequent addition of ryanodine, and in a timematched control bundle (fig. 2c). Note that in the example, the increased passive tension with theophylline (fig. 2b) appears similar to that induced by ryanodine (fig. 2a) but, in general, this effect was smaller and more variable with theophylline.

\section{Discussion}

This in vitro study demonstrated that in foreshortened canine diaphragm strips, theophylline-induced inotropic effects on twitch tension $(P \mathrm{t})$ were unaffected when transmembrane calcium fluxes were blocked either by verapamil $\left(10^{-5} \mathrm{M}\right)$ or by removing calcium from the extracellular level, even when buffered with EGTA (2 mM). In contrast, these inotropic effects were dependent on calcium release from sarcoplasmic reticulum as theophylline effects were abolished after ryanodine $\left(10^{-6} \mathrm{M}\right)$ pretreatment.

The model used in the present study was quite stable, as demonstrated by the results obtained in time-matched control experiments. Indeed, compared to initial values, the decrease in $P$ t over time averaged $-12 \pm 9 \%$ after 45 min (for all the protocols except those with ryanodine) and $-12 \pm 14 \%$ after $70 \mathrm{~min}$ (for ryanodine protocols).

Several attempts have been made to investigate the mechanism by which theophylline exerts its inotropic effects. These studies have suggested that theophylline induced a hyperpolarization of the cell membrane [11, 12], or interacted with intracellular [13-15] or transmembrane $[15,16]$ calcium transfer but not with cyclic adenosine monophosphate (cAMP) [15]. However, it should be noticed that all these experiments pertain to studies performed at optimal diaphragm length in rodents [11-16]. But, it is well-established that muscle mechanics at optimal length and at shorter length are different. Indeed, whilst tension generation decreases with muscle shortening due to a reduction in overlap between actin and myosin filaments [3] and due to calcium "deactivation" $[4,5]$, tension generation at optimal muscle length is maximal because filament geometry is optimal and calcium activation normal.

In our study, we attempted to investigate the mechanism involved in the preferential effect of theophylline at shorter length. This mechanism has never previously been explored. Thus, in view of previous reports implicating calcium deactivation in acute muscle shortening $[4,5]$, we examined the effects of theophylline on $P \mathrm{t}$ of foreshortened diaphragm in the presence of different calcium inhibitors to block separately each pathway able to enhance intracellular calcium level.

Firstly, theophylline's action was studied in the presence of a transmembrane block as it was proposed that diaphragmatic contraction at Lo was dependent on extracellular calcium [17]. Indeed, removal of calcium from the extracellular milieu or administration of verapamil, a voltage-dependent calcium channel blocker, significantly depressed muscle strength [18]. Although theophylline-induced inotropic effects at Lo have been demonstrated to depend on transmembrane calcium movement $[15,16,18,19]$, we could not observe a similar effect in foreshortened bundles. Indeed, calcium withdrawal from the extracellular milieu or the use of verapamil $\left(10^{-5} \mathrm{M}\right)$ did not affect $P \mathrm{t}$ of control bundles or the rise in $P \mathrm{t}$ induced by theophylline. Similar results were obtained in experiments where calcium-free solution was buffered with $2 \mathrm{mM}$ of EGTA. Note that, in these experiments, EGTA itself induced a depressant effect on $P \mathrm{t}$ of control bundles, a phenomenom observed previously [20] even with lower concentrations of EGTA [21]. Conversely, previous reports showed that the inhibitory effect of calcium withdrawal on muscle contraction resulted, in fact, from the absence of bound metal cations (like calcium) to the voltage sensor of the membrane [22, 23]; thereby abolishing its function in excitation-contraction coupling. This function was, however, restored when other metal cations (other than calcium) were added to the calcium-free solution. The absence of muscle contraction in calcium-free solution is, therefore, related more to inactivation of the voltage sensor in the absence of cations essential for its function rather than to the lack of a transmembrane calcium transient. 
From our above-mentioned experiments, it follows that theophylline-induced inotropic effects on foreshortened diaphragm were not related to transmembrane calcium flux. Therefore, the action of theophylline was studied when calcium release from the sarcoplasmic reticulum was blocked with ryanodine $\left(10^{-6} \mathrm{M}\right)$. Indeed, the calcium release channel is abundantly present in the terminal cisternae of skeletal sarcoplasmic reticulum. It there forms a homo-tetrameric complex with the foot structure, which spans the $12 \mathrm{~nm}$ gap between the Ttubules and the sarcoplasmic membranes [24, 25]. Following depolarization, this channel releases calcium from the sarcoplasmic reticulum in a still unelucidated way. In a complex manner, ryanodine binds to this release calcium channel at different sites in a micromolar range and, after an initial activation phase, ryanodine inactivates the calcium release channel [26].

After ryanodine pretreatment, $P \mathrm{t}$ was similarly depressed in control and experimental bundles and half-relaxation time similarly prolonged. Theophylline, subsequently added, failed to increase $P$ t. An even more dramatic depression of $P \mathrm{t}$ after ryanodine treatment was previously observed in rat diaphragm [8]. In fact, this effect referred to ryanodine action on calcium channel conductance. Indeed, ryanodine progressively locked the sarcoplasmic reticulum calcium channels in an open subconductance state, such that $P$ t decreased as a result of a decrease in the amount of calcium available for release [27]. As a consequence, a passive leak of calcium occurs, thereby causing muscle contraction [9], a phenomenon also obtained in our study. Nevertheless, to ensure that a toxic effect of ryanodine would have masked theophylline-induced inotropic effects, in another set of experiments, theophylline was added before ryanodine (experimental bundles) and these data were compared to time-matched control bundles. Under these circumstances, the depression in $P \mathrm{t}$ of experimental bundles and time matched control bundles was small and similar, thereby excluding a nonspecific effect of ryanodine.

The theophylline-induced inotropic effects on foreshortened diaphragm may be clinically relevant. Indeed, as respiratory muscles shorten with acute hyperinflation [28], they may become considerably more susceptible to the inotropic action of theophylline, such that inotropic actions may be present at considerably lower serum levels. Whether this actually occurs in these patients and whether or not it affects clinical outcome variables has not been studied. Clinical trials comparing the effects of theophylline in patients with or without acute hyperinflation appear warranted.

In conclusion, the present study demonstrated that the inotropic effects of theophylline on $P \mathrm{t}$ of foreshortened canine diaphragm bundles were not related to extracellular calcium but were essentially dependent on the release of calcium from the sarcoplasmic reticulum. This effect could result either from a direct action of theophylline on sarcoplasmic calcium channels or from interaction with an upstream event in the excitation-contraction coupling chain of skeletal muscle.

Acknowledgements: The authors thank N. Buts for her expert technical help.

\section{References}

1. Gayan-Ramirez G, Palecek F, Chen Y, Janssens S, Decramer M. Inotropic effects of aminophylline on canine diaphragm are enhanced by hyperinflation. J Appl Physiol 1994; 76: 39-44.

2. Gayan-Ramirez G, Decramer M. Inotropic effects of theophylline on foreshortened canine diaphragm. Am J Respir Crit Care Med 1994; 149: 920-924.

3. Gordon AM, Huxley AF, Julien FJ. The variation in isometric tension with sarcomere length in vertebrate muscle fibers. J Physiol (Lond) 1966; 184: 170-192.

4. Gonzalez-Serratos H, Somlyo AV, McClellan G, Shuman $\mathrm{H}$, Borrero LM, Somlyo AP. Composition of vacuoles and sarcoplasmic reticulum in fatigued muscle: electron probe analysis. Proc Natl Acad Sci USA 1978; 75: 1329-1333.

5. Ridgway EB, Gordon AM. Muscle activation: effects of small length changes on calcium release in single fibers. Science 1975; 189: 881-884.

6. Taylor SR, Rüdel R. Striated muscle fibers: inactivation of contraction induced by shortening. Science 1970; 167: $882-884$.

7. Lopez JR, Wanek LA, Taylor SR. Skeletal muscle: length-dependent effects of potentiating agents. Science 1981; 214: 79-82.

8. Herve P, Lecarpentier Y, Brenot F, Clergue M, Chemla $\mathrm{D}$, Duroux P. Relaxation of the diaphragm muscle: influence of ryanodine and fatigue. J Appl Physiol 1988; 65: 1950-1956.

9. Zavecz JH, Anderson W. Role of extracellular $\mathrm{Ca}^{2+}$ in diaphragmatic contraction: effects of ouabain, monensin and ryanodine. J Appl Physiol 1992; 73: 30-35.

10. McCully KK, Faulkner JA. Length-tension relationship of mammalian diaphragm muscles. J Appl Physiol: Respirat Environ Exercise Physiol 1983; 54: 16811686.

11. Esau SA. Effect of theophylline on membrane potential and contractile force in hamster diaphragm in vitro. J Clin Invest 1986; 77: 638-640.

12. Delbono O, Kotsias BA. Hyperpolarizing effect of aminophylline, theophylline and cAMP on rat diaphragm fibers. J Appl Physiol 1988; 64: 1893-1899.

13. Aubier M, Roussos C. Effect of theophylline on respiratory muscle function. Chest 1985; 88: 91S-97S.

14. Aubier M. Effect of theophylline on diaphragmatic and other skeletal muscle function. J Allergy Clin Immunol 1986; 78: 787-792.

15. Kolbeck RC, Speir WA. Theophylline, fatigue, and diaphragm contractility: cellular levels of $45 \mathrm{Ca}$ and cAMP. J Appl Physiol 1991; 70: 1933-1937.

16. Aubier M, Murciano D, Viires N, Lecocguic Y, Pariente R. Diaphragmatic contractility enhanced by aminophylline: role of extracellular calcium. J Appl Physiol: Respirat Environ Exercise Physiol 1983; 54: 460-464.

17. Viires N, Murciano D, Seta JP, Dureuil B, Pariente R, Aubier M. Effects of $\mathrm{Ca}^{2+}$ withdrawal on diaphragmatic fiber tension generation. J Appl Physiol 1988; 64: 26-30.

18. Kolbeck RC, Speir WA. Diltiazem, verapamil, and nifedipine inhibit theophylline-enhanced diaphragmatic contractility. Am Rev Respir Dis 1989; 139: 139-145.

19. Esau SA. Interaction of theophylline, verapamil, and diltiazem on hamster diaphragm muscle force in vitro. Am J Physiol 1988; 254: C365-C371.

20. Armstrong CM, Bezanilla FM, Horowicz P. Twitches in the presence of ethylene glycol bis( $\beta$-amino-ethyl 
ether)-N, N, N', N'-tetra-acetic acid. Biochim Biophys Acta 1972; 267: 605-608.

21. Bikhazi GB, Flores C, Foldes FF. The effect of verapamil and EGTA on the rat phrenic nerve-hemidiaphragm preparation. Anesth Analg 1985; 64: 505-508.

22. Brum G, Fitts R, Pizarro G, Rios E. Voltage sensors of the frog skeletal muscle membrane require calcium to function in excitation-contraction coupling. J Physiol (Lond) 1988; 398: 475-505.

23. Pizarro G, Fitts R, Uribe I, Rios E. The voltage sensor of excitation-contraction coupling in skeletal muscle. $J$ Gen Physiol 1989; 94: 405-428.

24. Inui M, Saito A, Fleischer S. Purification of the ryanodine receptor and identity with feet structures of junctional terminal cisternae of sarcoplasmic reticulum from fast skeletal muscle. J Biol Chem 1987; 262: 17401747.

25. Lai FA, Meissner G. The muscle ryanodine receptor and its intrinsic $\mathrm{Ca}^{2+}$ channel activity. J Bioenerg Biomemb 1989; 21: 227-246.

26. Fleischer S, Ogunbunmi EM, Dixon MC, Fleer EAM. Localization of $\mathrm{Ca}^{2+}$ release channels with ryanodine in junctional terminal cisternae of sarcoplasmic reticulum of fast skeletal muscle. Proc Natl Acad Sci USA 1985; 82: 7256-7259.

27. Fryer MW, Lamb GD, Neering IR. The action of ryanodine on rat fast and slow intact skeletal muscles. $J$ Physiol (Lond) 1989; 414: 399-413.

28. Decramer M. Effects of hyperinflation on the respiratory muscles. Eur Respir J 1989; 2: 299-302. 